\title{
ASSOCIATION OF PSYCHOLOGICAL STATUS WITH FIRST DIAGNOSED PULMONARY TUBERCULOSIS IN MEN
}

\author{
Maryna Kochuieva \\ Department of Internal Medicine ${ }^{2}$ \\ kochuevamarina@gmail.com \\ Vasyl Kushnir \\ Department of Phthisiology, Pulmonology and Family Medicine ${ }^{l}$ \\ Department of General and Clinical Immunology and Allergology ${ }^{2}$ \\ dikaryok@gmail.com \\ Ivan Hrek \\ Department of Phthisiology, Pulmonology and Family Medicine $e^{l}$ \\ Department of General and Clinical Immunology and Allergology ${ }^{2}$ \\ grek.ivan.md@gmail.com \\ ${ }^{1}$ Kharkiv Medical Academy of Postgraduate Education \\ 58 Amosova str., Kharkiv, Ukraine, 61176 \\ ${ }^{2}$ V. N. Karazin Kharkiv National University \\ 4 Svobody sq., Kharkiv, Ukraine, 61022
}

\begin{abstract}
The aim. Assessment of the initial psychological status of men with first diagnosed pulmonary tuberculosis and the study of its associations with the clinical course of the disease.

Materials and methods. The study involved 54 men with first diagnosed pulmonary tuberculosis. Patients had a dynamic complex clinical, laboratory and instrumental examination with an assessment of the psychological status according to the StateTrait Anxiety Inventory (STAI) and the Beck Depression Inventory (BDI).

Results. The evaluating of initial psychological status in patients with first diagnosed pulmonary tuberculosis, depressive states of varying severity were found in $47.05 \%$ of patients, moderate state anxiety - in $74.51 \%$ of patients, moderate trait anxiety in $35.29 \%$. We revealed associations of the levels of anxiety and depression with the degree of bacterial excretion and the duration of the intensive phase of therapy.

Conclusions. To assess the psychological status of patients with first diagnosed infiltrative pulmonary tuberculosis, it is advisable to use the STAI and the BDI.

Patients with first diagnosed infiltrative pulmonary tuberculosis in $100 \%$ of cases have psychological status disorders with a predominance of moderate state anxiety and the absence of depressive disorders in only half of them. More expressed violations of the psychological status (anxiety, depression) identifies patients who are married and have a steady job.

In patients with first diagnosed infiltrative pulmonary tuberculosis, an increase in the severity of anxiety and depression is associated with more expressed bacterial excretion, decrease in body weight, greater frequency of destruction of lung tissue, prevalence of the tuberculosis process by more than 2 lung segments, decrease in the effectiveness of standard therapy and prolongation intensive phase of treatment.
\end{abstract}

Keywords: infiltrative pulmonary tuberculosis, lung destruction, depression, anxiety, psychological status, efficiency of treatment, psychological sphere, STAI, BDI, marital status.

DOI: $10.21303 / 2504-5679.2021 .001800$

\section{Introduction}

According to World Health Organization's report 2019, tuberculosis has caused an estimated 1.4 million deaths. At the same time, over the same period, about 10 million new cases of the disease were detected. Despite the fact that over the past 20 years, modern medicine has managed to save more than 60 million people from tuberculosis, the problem still does not lose its relevance and requires targeted attention from the state and the medical community [1]. Today tuberculosis 
is epidemic in nature, and the fight against it is aimed at solving the most important medical and social problem [2].

Among the factors that can influence the development and course of tuberculosis infection, the psychological state of the patient may have a certain value [3].

According to the literature, there are common risk factors for the development of tuberculosis and depression $[4,5]$. It is also assumed that the chances of developing tuberculosis are significantly increased in patients with depression at the background of a decrease of immune defences [6]. It is known that with the background of depression there is a suppression of cellular immunity factors activity and an increase of catecholamines, histamine, opioids and some pro-inflammatory cytokines expression, which negatively affect the susceptibility to tuberculosis infection [7]. In turn, the chronicity of the tuberculous process causes the progression of tissue hypoxia, contributing to the development of anxiety and depression [8].

With the depression of psychological state in tuberculous patients, cases of alcohol and drug abuse are increasing. This is negatively affects adherence to treatment, increases the likelihood of developing drug resistance of Mycobacterium tuberculosis and prolongs the period of bacterial excretion [9].

Compared to healthy people, the prevalence of depression in tuberculosis patients is 3 times higher $[10,11]$. Age is of great importance, so in patients over 35 years of age, the risk of developing depression reaches $40-70 \%[12,13]$, and after 65 years, this index becomes even higher [14]. In countries with low developed economies the prevalence of depression among patients with tuberculosis is in the range $46-80 \%$ [15].

Most of modern research studied disorders of the psychological status in patients with diabetes mellitus, malignant tumours, ischemic heart disease, COPD [16, 17]. At the same time, there are few studies of psychological characteristics in patients with pulmonary tuberculosis. This was the reason for our research.

The aim of our study was to assess the initial psychological status of men with first diagnosed pulmonary tuberculosis and the study of its associations with the clinical course of the disease.

\section{Materials and methods}

The results of the research was conducted on the basis of: Municipal non-profit enterprise of Kharkiv regional council «Regional anti-tuberculosis dispensary No. 1», Municipal non-profit enterprise of Kharkiv regional council «Regional tuberculosis hospital No. 1», State health institution Regional tuberculosis 3, Municipal non-profit enterprise of Kharkiv regional council «Regional clinical anti-tuberculosis dispensary No. 7». The study was conducted in the period 2020-2021.

The study included 51 patients, all men with first diagnosed infiltrative pulmonary tuberculosis aged 25 to 50 years (mean age $40 \pm 7.76$ years) without concomitant diseases.

The patients had a comprehensive study of clinical, laboratory and instrumental parameters. The examination and treatment were carried out in accordance with the current protocol of the Ministry of Health of Ukraine No. 620 «Tuberculosis». All patients were questioned using the Spielberger's Anxiety STAI (State-Trait Anxiety Inventory) [18] adapted by Khanin Y. (1978) [19] and the Beck Depression Inventory (BDI) [20].

The study was conducted in accordance with the requirements of good clinical practice, the Council of Europe Convention on Human Rights and Biomedicine, the Helsinki Declaration of the World Medical Association and approved by the local ethics committee of the Kharkiv Medical Academy of Postgraduate Education (protocol No. 1, from 11.02.2021). All patients signed informed consent for participating in the clinical trial.

Statistical analysis of the results was carried out using the Microsoft Excel 9 software. We used descriptive statistics throughout the study. We used the Mann-Whitney U-test to validate the study.

\section{Results}

We examined 51 patients with first diagnosed infiltrative pulmonary tuberculosis. According to the results of a survey on the STAI, it was found that the proportion of patients with low state anxiety (S-Anxiety) was $25.49 \pm 6 \%(n=13)$. The remaining $74.51 \pm 6 \%$ of the study participants $(n=38)$ 
had a moderate S-anxiety. The proportion of patients with low trait anxiety (T-Anxiety) was $64.71 \pm 7 \%(n=33)$, and with moderate T-anxiety $-35.29 \pm 7 \%(n=18)$.

After questioning patients on the BDI, it was found that $52.94 \pm 7 \%$ of patients $(n=27)$ had no signs of depressive disorders, and $37.25 \pm 7 \%(n=19)$ and $9.8 \pm 4 \%(n=5)$ had respectively moderate and mild depression.

In $58.82 \pm 7 \%$ of patients $(n=30)$, sputum microscopy showed massive MTB excretion. All of these patients had moderate S-anxiety. Among patients with moderate T-anxiety, the proportion of massive MTB-excretors was $88.89 \pm 8 \%(n=16)$, and among patients with low T-anxiety $42.42 \pm 9 \%(n=14)$.

All patients with moderate depression was in the group of massive MTB excretion, while in patients with mild depression and without depression, massive bacterial excretion was detected, respectively, in $63.16 \pm 11 \%(n=12)$ and $48.15 \pm 10 \%(n=13)$ cases.

Patients with moderate S-anxiety in $78.95 \pm 7 \%(n=30)$ of cases had destructive changes in the lungs, and patients with a low S-anxiety - in $7.69 \pm 8 \%(n=1)$. In patients with moderate and low T-anxiety decay cavities in the lungs were detected in $88.89 \pm 8 \%(n=16)$ and $45.45 \pm 9 \%(n=15)$ cases respectively.

All men with moderate depression belonged to group with destructive changes in the lung tissue. In patients with mild depression decay cavities were found in $84.21 \pm 9 \%(n=16)$ and in patients without depression in $37.04 \pm 9 \%(n=10)$ cases.

The prevalence of tuberculous process over 2 segments of the lungs was detected in $52.63 \pm 8 \%$ of patients $(n=20)$ with a moderate S-anxiety, in $15.38 \pm 10 \%$ of patients $(n=2)$ with a low S-anxiety, in $77.78 \pm 11 \%(n=14)$ among patients with a moderate T-anxiety and in $24.24 \pm 7.5 \%(n=8)$ with a low T-anxiety.

All patients with moderate depression had pulmonary tissue damage in more than 2 segments, while in patients with mild depression and without depression, the prevalence of lung damage in more than 2 segments was in $63.16 \pm 11 \%(n=12)$ and $18.52 \pm 8 \%(n=5)$ cases respectively.

We studied the features of the psychological status depending on marriage, the presence of a permanent job, as well as the relationship between the severity of psychological disorders and the patient's body weight. The distribution of S-anxiety, T-anxiety levels and the severity of depression in the examined patients depending on marriage shown at Fig. 1.

Reducing of body mass index (BMI) was found in $39.47 \pm 8 \%$ of patients $(n=15)$ with a moderate S-anxiety and in $7.69 \pm 8 \%(n=1)$ with a low S-anxiety. Among the study participants with moderate and low T-anxiety reduction of BMI respectively found in $61.11 \pm 12 \%(n=11)$ and $15.15 \pm 6.5 \%(n=5)$ of cases.

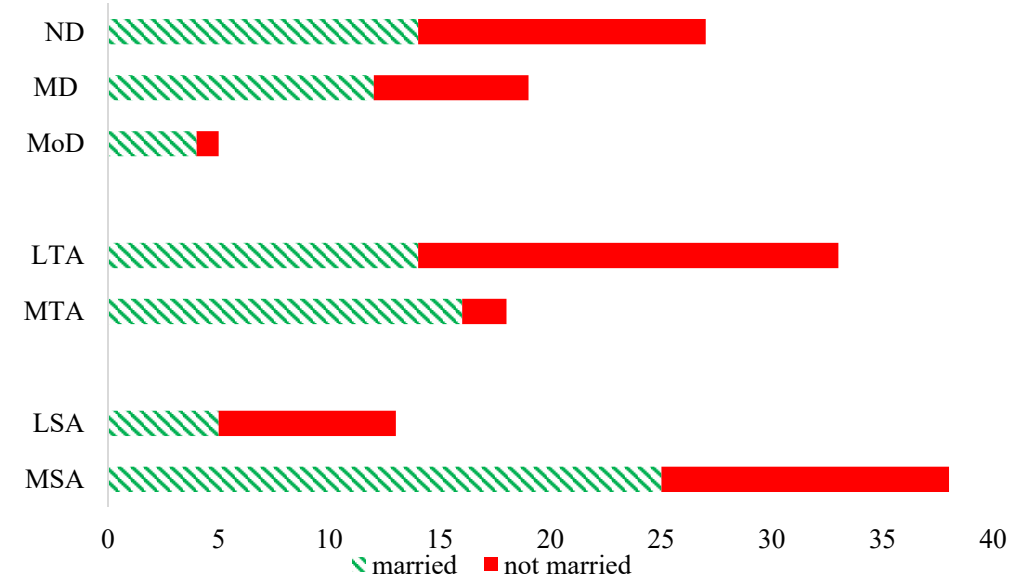

Fig. 1. S-anxiety, T-anxiety levels and the severity of depression in men with first diagnosed pulmonary tuberculosis, depending on marriage. (ND - no depression, MD - mild depression, MoD - moderate depression, LTA - low T-anxiety, MTA - moderate T-anxiety, LSA - low S-anxiety, MSA - moderate S-anxiety) 
All patients with moderate depression were included in the group with a reduced BMI. Among patients with mild depression reduction in BMI was found in $42.1 \pm 12 \%$ of cases $(n=8)$, and in patients without depression $-11.11 \pm 6 \%(n=3)$.

T-anxiety, S-anxiety levels of and the degree of depression in patients depending by employment shown at Fig. 2.

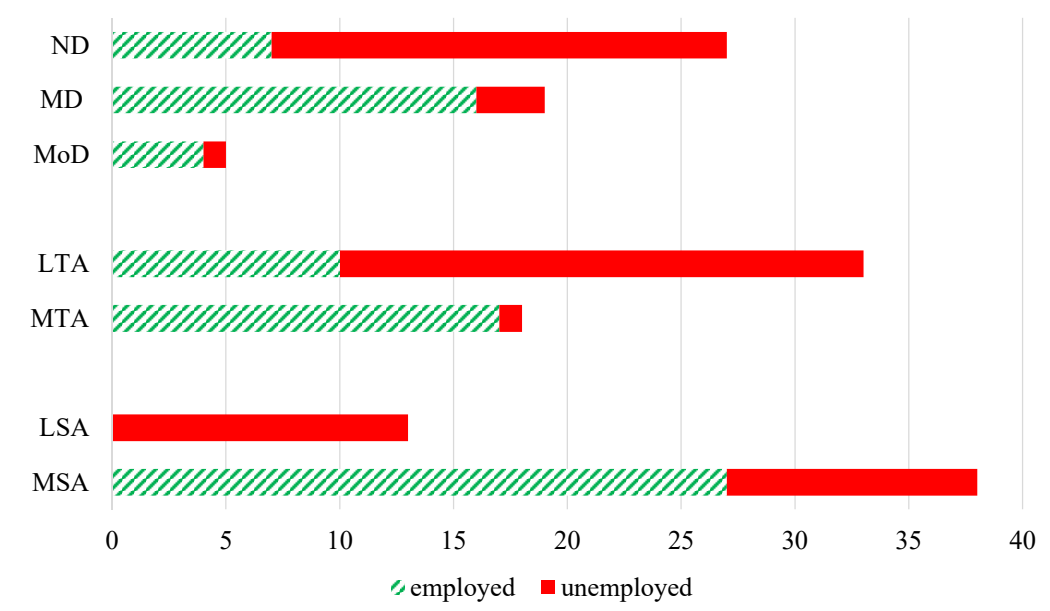

Fig. 2. T-anxiety, S-anxiety levels and the degree of depression in patients depending on employment. (ND - no depression, MD - mild depression, MoD - moderate depression, LTA - low T-anxiety, MTA - moderate T-anxiety, LSA - low S-anxiety, MSA - moderate S-anxiety)

Patients with a low S-anxiety did not require prolongation of therapy, while $36.84 \pm 8 \%$ of patients $(n=14)$ with a moderate S-anxiety demanded prolongation of treatment for 30 days. Among men with a moderate T-anxiety prolongation of treatment took place in $55.55 \pm 12 \%$ of cases $(n=10)$, and in patients with a low T-anxiety - in $12.12 \pm 6 \%(n=4)$.

All men with moderate depression required a 30-day prolongation of therapy. In $36.84 \pm 11 \%$ of cases $(n=7)$ treatment was prolonged in patients with mild depression and in $7.4 \pm 5 \%$ of cases $(n=2)$ in patients without depression.

\section{Discussion}

The results of our study showed the effectiveness and appropriateness of questioning patients with first diagnosed pulmonary tuberculosis using the STAI and BDI. According to the questionnaire data, the patients were distributed as follows: according to the STAI, low S-anxiety was found in $25.49 \%(n=13)$ patients and $74.51 \%$ of patients $(n=38)$ showed moderate S-anxiety; low T-anxiety was found in $64.71 \%(n=33)$ patients, moderate T-anxiety - in $35.29 \%(n=18)$ patients. After responded patients on the BDI $52.94 \%(n=27)$ of them were assigned to the group without signs of depressive disorders and $37.25 \%(n=19)$ and $9.8 \%(n=5)$ respectively showed signs of moderate and mild depression. Severe anxiety and depression were not revealed in the observation group. The examined category of patients with pulmonary tuberculosis was characterized by the presence of moderate S-anxiety in 3/4 patients, and moderate T-anxiety and moderate depression in more than a $30 \%$ of patients. The results of the study indicated the presence of anxiety in $100 \%$ of patients with first diagnosed infiltrative pulmonary tuberculosis and the absence of depressive disorders in only half of them.

More than half of the patients $(58.82 \%)$ had massive MTB excretion according to sputum microscopy; all of them were in moderate S-anxiety group. In the group with moderate T-anxiety there were two times more massive MTB-excretors, than among patients with a low T-anxiety $(p=0.096)$. The severity of depressive disorders also showed a relationship with the massiveness of MTB excretion - with an increase in the severity of depression, the frequency of detecting massive MTB excretion also increased. Greater reductions in BMI also took place in patients with more severe 
disorders of psychological status. These results indicated the association of an increase in the severity of anxiety and depression with massive MTB excretion and weight loss in patients with first diagnosed infiltrative pulmonary tuberculosis.

The results of the study showed that destructive changes in the lungs, respectively, were $10(p<0.03)$ and $2(p=0.041)$ times more often in patients with a moderate S-anxiety and T-anxiety compared with their frequency in patients with mild forms of S-anxiety and T-anxiety. According to our study, destructive changes in the lungs were detected in all patients with moderate depression, in $84.21 \%$ of patients with mild depression, and in $37.04 \%$ of patients without depression.

The prevalence of the tuberculous process in more than two segments of the lungs, respectively, $3.4(p=0.018)$ and $3.2(p=0.002)$ times often in moderate S-anxiety and T-anxiety cases compared with their frequency in mild forms of anxiety. Patients with moderate depression in $100 \%$ of cases had lung damage over 2 segments, and patients with mild depression and without depression $-1.6(p>0.05)$ times and $5.4(p=0.053)$ times rarely, respectively. Thus, in patients with more symptoms of anxiety and depression, destructive and widespread lung lesions were revealed.

Our data also indicated that more expressed disorders of the psychological status (anxiety, depression) were detected in patients who were married and had a permanent job.

The prolongation of the intensive phase of treatment required $38.84 \%$ of patients with a moderate S-anxiety, $50 \%$ of patients with a moderate T-anxiety, $12.12 \%$ of patients with a low T-anxiety, all patients with moderate depression and, respectively, $36.84 \%$ and $7.4 \%$ of patients with mild depression and without depression. These data testified to the lesser effectiveness of standard therapy in more severe disorders of psychological status.

As shown in studies by Duko B., depression and anxiety in TB patients were detected in $43.4 \%$ and $41.5 \%$, respectively, which is similar to our study.

As shown in studies by Duko B. et al. depression and anxiety in TB patients were detected in $43.4 \%$ and $41.5 \%$, respectively, which is similar to our study [21]. In the study by Aamir S. et al. psychological disorders were observed in $72 \%$ of TB cases, which is much more [22]. Tola H. H. et al. in their study note a significant link between marital status and depression [23]. This manifests itself in increased rates of depression in unmarried patients. In our study, a different picture is observed with the prevalence of depression in married men. This may be due to the small sample of patients. This study showed an association of depression with less effective TB therapy, which is also reflected in a systematic review and meta-analysis in 2020 [24].

As noted in the Chandra M. study, depression remains a poor predictor of tuberculosis [25]. The combination of depressive disorders and tuberculosis requires joint approaches at the state level to develop a program for the management of such clinical cases.

The limitations of the research include the study of only men, a small number of patients, the use of only two questionnaires, the use of descriptive statistics.

In the future, the research should be continued in a larger number of patients, including women. In addition, it should include a questionnaire on quality of life, CT of the lungs, pulmonary spirometry and a study of indicators of humoral and cellular immunity.

\section{Conclusions}

It is advisable to use the STAI and the BDI to assess the psychological status of patients with first diagnosed infiltrative pulmonary tuberculosis.

Patients with first diagnosed infiltrative pulmonary tuberculosis in $100 \%$ of cases have psychological status disorders with a predominance of moderate S-anxiety and the absence of depressive disorders in only half of them. More heavily violations of the psychological status (anxiety, depression) identifies patients who are married and have a permanent job.

In patients with first diagnosed infiltrative pulmonary tuberculosis, an increase in the severity of anxiety and depression is associated with massive bacterial excretion, decrease in body weight, and greater frequency of lung tissue destruction, prevalence of the tuberculosis process by more than 2 lung segments, decrease in the effectiveness of standard therapy and prolongation intensive phase of treatment. 


\section{Conflict of interests}

The authors declare that they have no conflict of interests.

\section{Acknowledgments}

We would like to express our gratitude to the administration of the medical and prophylactic institutions who agreed to conduct the study.

\section{References}

[1] Global Tuberculosis Report (2020). World Health Organization. Licence: CC BY-NC-SA 3.0 IGO. Geneva, 232.

[2] Pezzella, A. T. (2019). History of Pulmonary Tuberculosis. Thoracic Surgery Clinics, 29 (1), 1-17. doi: http://doi.org/10.1016/ j.thorsurg.2018.09.002

[3] Courtwright, A., Turner, A. N. (2010). Tuberculosis and Stigmatization: Pathways and Interventions. Public Health Reports, 125 (4_suppl), 34-42. doi: http://doi.org/10.1177/00333549101250s407

[4] Shrestha, S. K., Joshi, S., Bhattarai, R. B., Joshi, L. R., Adhikari, N., Shrestha, S. K. et. al. (2020). Prevalence and risk factors of depression in patients with drug-resistant tuberculosis in Nepal: A cross-sectional study. Journal of Clinical Tuberculosis and Other Mycobacterial Diseases, 21, 100200. doi: http://doi.org/10.1016/j.jctube.2020.100200

[5] Dasa, T. T., Roba, A. A., Weldegebreal, F., Mesfin, F., Asfaw, A., Mitiku, H. et. al. (2019). Prevalence and associated factors of depression among tuberculosis patients in Eastern Ethiopia. BMC Psychiatry, 19 (1). doi: http://doi.org/10.1186/ s12888-019-2042-6

[6] Cho, I. Y., Chang, Y., Sung, E., Sohn, W., Kang, J.-H., Shin, H., Ryu, S. (2020). Depressive symptoms and risk of liver-related mortality in individuals with hepatitis B virus infection: a cohort study. Scientific Reports, 10 (1). doi: http://doi.org/10.1038/ s41598-020-77886-2

[7] Iftikhar, A., Islam, M., Shepherd, S., Jones, S., Ellis, I. (2021). Cancer and Stress: Does It Make a Difference to the Patient When These Two Challenges Collide? Cancers, 13 (2), 163. doi: http://doi.org/10.3390/cancers13020163

[8] Ehrt, S., Schnappinger, D., \& Rhee, K. Y. (2018). Metabolic principles of persistence and pathogenicity in Mycobacterium tuberculosis. Nature Reviews Microbiology, 16 (8), 496-507. doi: http://doi.org/10.1038/s41579-018-0013-4

[9] Dean, A. S., Cox, H., Zignol, M. (2017). Epidemiology of Drug-Resistant Tuberculosis. Strain Variation in the Mycobacterium Tuberculosis Complex: Its Role in Biology, Epidemiology and Control, 209-220. doi: http://doi.org/10.1007/978-3-319-64371-7_11

[10] Depression and Other Common Mental Disorders: Global Health Estimates (2017). World Health Organization. Available at: http://www.jstor.org/stable/resrep28026.1 Last accessed: 22.04.2021

[11] Picco, L., Subramaniam, M., Abdin, E., Vaingankar, J., Chong, S. (2017). Gender differences in major depressive disorder: findings from the Singapore Mental Health Study. Singapore Medical Journal, 58 (11), 649-655. doi: http://doi.org/10.11622/ smedj.2016144

[12] Sweetland, A., Oquendo, M., Wickramaratne, P., Weissman, M., Wainberg, M. (2014). Depression: a silent driver of the global tuberculosis epidemic. World Psychiatry, 13 (3), 325-326. doi: http://doi.org/10.1002/wps.20134

[13] Pardal, P., Singh, L., Prakash, J. (2015). Psychiatric morbidity in patients of pulmonary tuberculosis-an observational study. Industrial Psychiatry Journal, 24 (2), 168. doi: http://doi.org/10.4103/0972-6748.181722

[14] Wood, A. M., Boyce, C. J., Moore, S. C., Brown, G. D. A. (2012). An evolutionary based social rank explanation of why low income predicts mental distress: A 17 year cohort study of 30,000 people. Journal of Affective Disorders, 136 (3), $882-888$. doi: http://doi.org/10.1016/j.jad.2011.09.014

[15] Peltzer, K., Naidoo, P., Matseke, G., Louw, J., Mchunu, G., Tutshana, B. (2012). Prevalence of psychological distress and associated factors in tuberculosis patients in public primary care clinics in South Africa. BMC Psychiatry, 12 (1). doi: http://doi.org/10.1186/1471-244x-12-89

[16] Katon W. J. (2011). Epidemiology and treatment of depression in patients with chronic medical illness. Dialogues in clinical neuroscience, 13 (1), 7-23. doi: http://doi.org/10.31887/dens.2011.13.1/wkaton

[17] Almeida, S. S., Zizzi, F. B., Cattaneo, A., Comandini, A., Di Dato, G., Lubrano, E. et. al. (2020). Management and Treatment of Patients With Major Depressive Disorder and Chronic Diseases: A Multidisciplinary Approach. Frontiers in Psychology, 11. doi: http://doi.org/10.3389/fpsyg.2020.542444

[18] Spielberger, C. D., Gorsuch, R. L., Lushene, R. Vagg, P. R., Jacobs, G. A. (1983). Manual for the State-Trait Anxiety Inventory. Palo Alto: Consulting Psychologists Press.

[19] Karelin, A. (2007). Great Encyclopedia of Psychological Tests. Moscow: «Eksmo» Publishing House, 416.

[20] Beck, A. T., Steer, R. A., Carbin, M. G. (1988). Psychometric properties of the Beck Depression Inventory: Twenty-five years of evaluation. Clinical Psychology Review, 8 (1), 77-100. doi: http://doi.org/10.1016/0272-7358(88)90050-5 
[21] Duko, B., Gebeyehu, A., Ayano, G. (2015). Prevalence and correlates of depression and anxiety among patients with tuberculosis at WolaitaSodo University Hospital and Sodo Health Center, WolaitaSodo, South Ethiopia, Cross sectional study. BMC Psychiatry, 15 (1). doi: http://doi.org/10.1186/s12888-015-0598-3

[22] Aamir, S., Aisha (2010). Co-morbid anxiety and depression among pulmonary tuberculosis patients. Journal of the College of Physicians and Surgeons - Pakistan, 20 (10), 703-704.

[23] Tola, H. H., Shojaeizadeh, D., Garmaroudi, G., Tol, A., Yekaninejad, M. S., Ejeta, L. T. et. al. (2015). Psychological distress and its effect on tuberculosis treatment outcomes in Ethiopia. Global Health Action, 8 (1), 29019. doi: http://doi.org/ 10.3402/gha.v8.29019

[24] Ruiz-Grosso, P., Cachay, R., de la Flor, A., Schwalb, A., Ugarte-Gil, C. (2020). Association between tuberculosis and depression on negative outcomes of tuberculosis treatment: A systematic review and meta-analysis. PLOS ONE, 15 (1), e0227472. doi: http://doi.org/10.1371/journal.pone.0227472

[25] Chandra, M., Rana, P., Chandra, K., Arora, V. K. (2019). Tuberculosis - Depression syndemic: A public health challenge. Indian Journal of Tuberculosis, 66 (1), 197-202. doi: http://doi.org/10.1016/j.ijtb.2019.02.007

Received date 27.04.2021

Accepted date 17.05.2021

Published date 25.05.2021
(C) The Author(s) 2021

This is an open access article under the Creative Commons CC BY license

How to cite: Kochuieva, M., Kushnir, V., Hrek, I. (2021). Association of psychological status with first diagnosed pulmonary tuberculosis in men. EUREKA: Health Sciences, 3, 24-30. doi: http://doi.org/10.21303/2504-5679.2021.001800 